\title{
INFLUENCIA DEL CONTEXTO SOBRE EL CONOCIMIENTO DE LA FORMACIÓN PROFESIONAL DE LOS ESTUDIANTES DE EDUCACIÓN SECUNDARIA
}

\section{CONTEXT INFLUENCE ON THE VOCATIONAL TRAINING KNOWLEDGE OF SECONDARY SCHOOL PUPILS}

\author{
Susana Molina Martín* \\ Universidad de Oviedo
}

\section{RESUMEN}

En este artículo se analiza la información que sobre algunos aspectos de la formación profesional ocupacional tiene el alumnado que finaliza la educación obligatoria y reside en diversas ciudades asturianas. Los resultados obtenidos permiten concluir que su conocimiento no es muy amplío, siendo preciso desarrollar un sistema integrado y coordinado de orientación y formación profesional entre los centros educativos y la administración local.

Palabras-clave: Administración local, Orientación Profesional, Educación Secundaria, Coordinación de la educación, Ciudades educadoras.

\begin{abstract}
This paper analyzes the knowledge that students who are in the last year of compulsory secondary education, living in different cities of Asturias, have about several vocational training courses. The results obtained permit us to conclude that pupils do not have enough information. We considered that this situation would be able to change if both school centres and local government develop a proposal of vocational guidance and training together.

Key Words: Local government, Vocational Guidance, Secondary Education level, Educational coordination, Educating Cities.

\footnotetext{
* Profesora del Departamento de Ciencias de la Educación de la Universidad de Oviedo. Licenciada en Filosofía y Ciencias de la Educación y Doctora en Pedagogía. Miembro del grupo de investigación de la Universidad de Oviedo denominado ASOCED - Análisis Sociológico y Cultural de los Procesos Escolares y Educativos-, con la página web: http://www.uniovi.es/asoced. Facultad de Ciencias de la Educación, C/Aniceto Sela, s/n. Despacho 315.33005 - Oviedo. Correo-E: smmolina@uniovi.es
} 


\section{Introducción}

Todo ser humano está íntimamente ligado a un entorno. En éste, la persona no permanece ajena a lo que acontece a su alrededor, sino que es un ser activo en sus relaciones con el medio, lo que favorece su desarrollo y aprendizaje. Esta afirmación no es nueva, si tenemos en cuenta que en las civilizaciones clásicas, como en el caso de la griega y la romana, organizadas en torno a las ciudades, denominadas polis y urbs respectivamente, adquiere relevancia el entorno, en ese caso la ciudad, en la educación. Ciudad, que concibieron, como una sociedad educativa total, en la que toda la comunidad entera, con los jóvenes y los ancianos, en el ágora y en la palestra, a través de las costumbres y de las leyes, por el juego, el trabajo y el estudio, constituía un complejo entramado de influencias educadoras (Jaeguer, 1957; Bowen, 1976).

Si bien es cierto que las influencias educativas en la ciudad se producen simplemente por el sistema de relaciones y formas de vida que en ella se desarrollan, también lo es que en la actualidad las poblaciones poseen centros especializados para impartir la educación formal. No obstante, la complejidad del entorno contribuye a que la acción educativa no acabe ahí. Surgen necesidades educativas que no son cubiertas, que salen al escenario social y que demandan de la actuación de otros agentes. Uno de ellos es la administración local.

La ampliación de competencias y funciones, una mayor libertad a la hora de definir una política propia, junto al incremento de los recursos con lo que cuenta la Administración local, son consecuencia del agrupamiento de un número elevado de personas en un mismo territorio, a la vez que aspectos fundamentales para que adquiera un mayor protagonismo en la prestación de servicios dirigidos a satisfacer distintas necesidades de la población. E igual como sucede con otros ámbitos (salud, abastecimiento, ocio y tiempo libre, cultura, etc.), han comenzado a responsabilizarse y a desarrollar una función educativa (Molina, 2006) ${ }^{1}$.

Tras asumir la responsabilidad, surge la inevitable reflexión y la investigación centrada en determinar aquellos ámbitos educativos que atestiguan la existencia de necesidades de la ciudadanía que asoman al escenario social. Es posible que no haya existido nunca una atención a algunos de estos ámbitos, o que aún habiéndola no sea la más adecuada.

Porque surge la necesidad de trabajar $^{2}$ en un escenario complejo, el ser humano se encuentra con dificultades. Desempleo, cambios en la estructura del empleo, desplazamiento de la actividad remunerada al sector servicios (Castells, 1995), transformaciones en los perfiles de aptitudes, capacidades y experiencia que se precisan para entrar en el mercado laboral (Bell, 1989), incremento de la demanda de profesionales cualificados (Comisión Euro-

1. La educación como ámbito de la vida humana sobre el que se debe intervenir, es reconocido en documentos internacionales como la Declaración Universal de Derechos Humanos (Organización de Naciones Unidas, 1948), el Informe sobre la Educación de la UNESCO titulado Aprender a ser y dirigido por Faure (1973), la Carta de los Derechos Fundamentales de la Unión Europea (Consejo de la Unión Europa, 2001), la Carta Europea de salvaguarda de los Derechos Humanos en la Ciudad (Organización de Naciones Unidas, 2000), y la Carta Mundial por el Derecho a la Ciudad (Foro Social Mundial, 2005).

2. Uno de los momentos clave para comprender esta situación, es la profunda transformación que se produjo durante la Revolución Industrial, momento desde el cual, sólo unos pocos poseen los medios de producción, mientras la mayor parte de la población deja de poseer los bienes sustanciales para vivir, convirtiéndose el trabajo en una necesidad (Bell, 1989). 
pea, 1996; 1997), entre otros, son ejemplos significativos de cómo las experiencias del ser humano en relación con el trabajo, son completamente distintas de las vividas por generaciones anteriores.

La demanda de educación para afrontar los retos que se derivan del ámbito del trabajo es, por tanto, viva y actual para una ciudadanía ubicada en un entorno laboral sometido a un proceso de cambio continuado. Le interesará además de contar con un proyecto de formación, disponer de unos servicios de orientación para el desarrollo y ajuste del proyecto personal de inserción profesional. Se trata de establecer un sistema integrado de formación y orientación profesional con la finalidad de facilitar el empleo y el progreso de las personas a lo largo de su vida. Ciertamente, esta demanda supone ampliar los destinatarios de la acción, por ejemplo, al alumnado del sistema educativo y a sus familias, a los trabajadores desempleados, a los trabajadores ocupados, a las empresas, a los centros formativos, y a la sociedad en general (Ministerio de Trabajo y Asuntos Sociales, 2004a).

A la ciudad misma se le exige un compromiso, una intervención directa, al tratarse de una demanda de la ciudadanía. Bastarían algunos ejemplos para dejar constancia de la respuesta, hoy día, hacia la formación y orientación profesional desarrollada en el seno de algunas ciudades. En ciudades europeas, como Turín, el Ayuntamiento elabora el proyecto SCUO/LAV, que desarrolla en colaboración con la Cámara de Comercio, las asociaciones de emprendedores y las escuelas de educación secundaria. Se trata de una iniciativa que surge con el fin de introducir a los estudiantes en el mundo laboral, ofrecer orientación profesional durante los estudios y posibilitar el contacto entre las empresas y los jóvenes (Bonino, 2005) ${ }^{3}$. En el contexto asturiano, el Ayuntamiento de Oviedo cuenta con la denominada Agencia de Desarrollo Local, servicio dirigido tanto al desarrollo y fomento del empleo, como a la formación ocupacional. El Ayuntamiento de Gijón aglutina políticas de empleo, formación, comercio y turismo, promoción económica e industrial e innovación tecnológica y organizativa en la Agencia Local de Promoción Económica y Empleo. Al igual que los anteriores, también el Ayuntamiento de Avilés cuenta con una Agencia de Desarrollo Local ${ }^{4}$.

Puede contemplarse, pues, la ciudad como un agente educativo que desarrolla su propia intervención en materia de formación y orientación profesional. Este artículo ofrece, por tanto, algunos resultados de un estudio que muestra de un modo indirecto los resultados de estas acciones. Su utilidad proviene de considerar al alumnado como la fuente de la que tratamos de obtener datos que amplíen la visión que tenemos de la actuación que se desarrolla desde algunos centros educativos en coordinación con el ayuntamiento. El conocimiento que tiene el alumnado que cursa cuarto de Educación Secundaria Obligatoria (E.S.O.), próximo a la toma de decisiones sobre su futuro profesional, constituye una aportación imprescindible. No se trata, en ningún caso, de sacar a la luz todos sus conocimientos, sino aquellos relacionados con programas de formación ocupacional, a los que estos estudiantes podrían optar al finalizar la E.S.O. por su mayor vinculación con los ayuntamientos.

3. En España, el Ayuntamiento de Sant Cugat del Vallés pone en marcha el denominado Dispositivo Local para la transición escuela-trabajo, órgano formado por diversos profesionales de la educación y de la administración local, y creado para llevar a cabo las actuaciones de apoyo social a la inserción y orientación laboral del alumnado del segundo ciclo de educación secundaria obligatoria, bachillerato y ciclos formativos (Ferrando, 2005).

4. La información disponible del Ayuntamiento de Oviedo puede consultarse en: http://www4.ayto-oviedo.es; del Ayuntamiento de Gijón en: http://www.ayto-gijon.es; y del Ayuntamiento de Avilés en: http://www.ayto-aviles.es 


\section{Método}

\section{Objetivos de la investigación}

Se pretende realizar un análisis del conocimiento que tiene el alumnado que finaliza la E.S.O. acerca de algunos programas formativos. Se trata de explorar los conocimientos de los estudiantes que viven en las tres grandes ciudades asturianas, que además se definen a sí mismas como educadoras (y por tanto, con un compromiso firme en la educación de sus ciudadanos $^{5}$ ), como son Gijón, Oviedo y Avilés, para comprobar si son diferentes o semejantes, tanto entre ellos, como de los que tienen otros estudiantes que residen en otras localidades más pequeñas en los que los ayuntamientos podrían contar con menos recursos para promover la formación y la orientación laboral. Concretamente los objetivos específicos que se pretenden explorar en el presente artículo son:

1. Determinar las diferencias y semejanzas que, en función del lugar de residencia ${ }^{6}$, se producen en el conocimiento que los estudiantes demuestran de: a) lugares donde aprender oficios distintos de los centros donde se imparte Educación Secundaria; b) becas para el aprendizaje de oficios.

2. Analizar las semejanzas y diferencias que, en función del lugar de residencia, se producen respecto al conocimiento que tienen de algunos programas formativos ocupacionales (Escuelas Taller, Casas de Oficios, Programas de Garantía Social y cursos del INEM), concretamente respecto a: a) su existencia; b) el conocimiento de personas que hayan participado en ellos; c) los estudios que se precisan para participar en ellos.

3. Estudiar la importancia que tiene para el alumnado a la hora de tomar decisiones sobre su futuro profesional la orientación que les proporcionan en el centro educativo.

\section{Instrumento de recogida de datos}

Con la finalidad de realizar el mencionado análisis, se utilizan los datos obtenidos a través de un cuestionario que tiene un objetivo más amplio: "obtener un diagnóstico de las expectativas de formación y empleo del alumnado de cuarto curso de E.S.O. en el Principado de Asturias" 7 . El cuestionario, en cuyo diseño se combinan preguntas de tipo abierto y cerrado, consta de 62 ítems que se pueden agrupar en cuatro amplios bloques ${ }^{8}$. No obstante, para realizar el análisis pretendido, se emplean ítems del último bloque, donde se les pregunta:

5. Los compromisos de las ciudades que se denominan a sí mismas educadoras, pueden consultarse en la página Web de la Asociación Internacional de Ciudades Educadoras: http://www.edcities.bcn.es

6. Con la expresión "lugar de residencia" me refiero a las tres ciudades abordadas (Gijón, Oviedo y Avilés), así como al resto de las localidades abordadas de forma conjunta.

7. Investigación financiada en el marco del Plan Nacional de Investigación Científica, Desarrollo e Innovación Tecnológica (2003-2006).

8. En un primer bloque, que consta de 18 ítems, se recogen los datos sociodemográficos de la muestra. En un segundo bloque, que abarca desde el ítem 19 al 37, se profundiza en la impresión del alumnado sobre la marcha del curso, así como en sus expectativas académicas. En un tercer bloque, que comprende desde el ítem 38 al 51 , se abordan las expectativas laborales. En el cuarto bloque, que incluye desde el ítem 52 al 62, nos centramos en su conocimiento y expectativas de formación-empleo en distintas ocupaciones. 
- ¿Conoces algún lugar dónde puedas aprender un oficio o profesión distinto a los centros en los que se estudia Bachillerato o Formación Profesional? (Sí/No). En caso afirmativo ¿cuál?

- ¿Crees que existen becas para aprender oficios? (Sí/No). En caso afirmativo ¿cuál?

- ¿Has oído hablar alguna vez de los siguientes programas? (Escuela Taller / Casas de Oficios / Programas de Garantía Social / Cursos del INEM). En caso afirmativo señala cuáles.

- ¿Conoces a alguien que haya participado en ellos? (Sí/No).

- ¿Qué estudios crees que se necesitan para participar en estos programas (ninguno, superar la ESO, Bachillerato o F.P., estudios universitarios, no lo sé)?

- ¿En relación con la definición de tu futuro profesional ¿cómo estás?

- Para decidir lo que vas a estudiar o en lo que vas a trabajar después de la ESO ¿qué importancia tienen para ti la orientación que me dan en el colegio (ninguna, poca, bastante o mucha)?

\section{Muestra}

Los criterios que se siguen para elaborar la muestra a la que aplicar el cuestionario son tanto estadísticos como estratégicos. Es preciso, por un lado, respetar la proporcionalidad de la densidad escolar de cada zona geográfica y, por otro, que determinadas zonas rurales sean estudiadas de forma representativa por sí mismas. A partir de aquí se tuvieron en cuenta los siguientes criterios muestrales: zonas geográficas en que está dividida Asturias (zona centro, cuencas, oriente y occidente), titularidad de los centros, comarcas escolares en que la Consejería de Educación tiene dividida la región, densidad escolar, $\mathrm{y}$, por último, relación rural y urbano. Sobre estos criterios se produce la afijación de cotas y, a partir de ahí, se refuerza la representación de algunas áreas rurales, con objeto de que sean estudiadas con verdadera representatividad.

La muestra participante la forman un total de 2.260 estudiantes de cuarto curso de E.S.O., con edades comprendidas entre catorce y dieciocho años. Concretamente, el alumnado residente en las tres localidades que se abordan, Oviedo (23,5\%), Gijón (21\%) y Avilés $(8,7 \%)$, supera la mitad del total de la muestra (1.203 estudiantes), lo que supone el 53,2\% ${ }^{9}$.

\section{Procedimiento}

La participación de los centros que imparten E.S.O. en el Principado de Asturias es voluntaria. El cuestionario se aplica en el curso 2005/06, en el horario regular de cada una de las clases por los miembros del grupo que llevan a cabo la investigación ${ }^{10}$, en una sesión de aproximadamente una hora de duración. Una vez que el alumnado contesta al cuestionario,

9. Las localidades de residencia seleccionadas, según la zona geográfica de Asturias, son: 1) Localidades centro: Avilés, Corvera, Gijón, Lugones, Noreña, Oviedo, Piedras Blancas, Pravia, Salinas, Siero. 2) Localidades cuencas: Entrego, La Felguera, Mieres, Sama, Ujo. 3) Localidades oriente: Arriendas, Cangas de Onís, Infiesto, Llanes, Luces, Villaviciosa. 4) Localidades occidente: Boal, Grandas de Salime, Grado, Luarca, Navia, Pola de Lena, San Antolín de Ibias, Salas, Tapia, Tineo, Vegadeo.

10. Grupo de investigación ASOCED (Análisis Sociológico y Cultural de los Procesos Escolares y Educativos) del Departamento de Ciencias de la Educación de la Universidad de Oviedo. 
se procede a la categorización de los datos obtenidos, utilizando como sistema de análisis estadístico y gestión de datos el programa SPSS.

\section{Resultados}

El alumnado que se encuentra en el último curso de E.S.O. muestra un gran desconocimiento de los programas formativos dirigidos al aprendizaje de oficios que se desarrollan en otros centros diferentes de aquellos en los que estudian Bachillerato y Formación Profesional. Como se puede observar en la tabla $1^{11}$, sólo el $23.6 \%$ afirma conocerlos. Respuesta que no está condicionada por el hecho de vivir en una u otra localidad, aunque las tres grandes ciudades asturianas abordadas muestran porcentajes de respuesta algo menores al porcentaje medio (23,6\%): $22.3 \%$ en Gijón, $21.3 \%$ en Oviedo y $21.8 \%$ en Avilés.

TABLA 1. Conocimiento de lugares dónde aprender un oficio.

\begin{tabular}{|c|c|c|c|c|c|c|c|}
\hline \multirow{3}{*}{$\begin{array}{l}\text { ¿Conoces } \\
\text { algún lugar...? } \\
\mathrm{Si}\end{array}$} & \multicolumn{6}{|c|}{ Localidades } & \multirow{3}{*}{$\begin{array}{c}\begin{array}{c}\text { Otras } \\
\text { Localidades }\end{array} \\
\chi \\
23.6 \%\end{array}$} \\
\hline & \multicolumn{2}{|c|}{ Avilés } & \multicolumn{2}{|c|}{ Gijón } & \multicolumn{2}{|c|}{ Oviedo } & \\
\hline & 43 & $21,8 \%$ & 106 & $22,3 \%$ & 113 & $21,3 \%$ & \\
\hline No & 140 & $71,1 \%$ & 356 & $74,9 \%$ & 404 & $76,1 \%$ & $73.4 \%$ \\
\hline NS/NC & 14 & $7,1 \%$ & 13 & $2,7 \%$ & 14 & $2,6 \%$ & $3 \%$ \\
\hline
\end{tabular}

Cuando se les pregunta por los lugares que afirman conocer, se produce una gran dispersión en las respuestas. Un 4\% del alumnado responde que conocen las Escuelas Taller, un $1.8 \%$ se refieren a las Casas de Oficios, un $0.3 \%$ aluden a los programas de Garantía Social, un $0.1 \%$ señala los cursos del INEM-FIP, mientras un $17.2 \%$ del total responde otras opciones, encontrándose entre las más representativas: academias, el aprendizaje directo con un profesional o en la empresa y FUCOMI ${ }^{12}$.

Respecto al conocimiento de la existencia de becas para aprender oficios, en la tabla 2 se puede observar que, en general, también se produce un gran desconocimiento, puesto que el $73.9 \%$ de los encuestados responde negativamente. En relación con las ciudades abordadas, únicamente Avilés muestra variaciones respecto a los porcentajes medios de las demás localidades, presentando diferencias estadísticamente significativas respecto a Gijón y Oviedo (Coeficiente de contingencia $=0.101 ; \mathrm{P}=0.015$ ). Así, el mayor desconocimiento de los estudiantes respecto a la existencia de becas se produce en Avilés, donde sólo en $13.7 \%$ responde afirmativamente. Por el contrario, en Oviedo y en Gijón los porcentajes de respuesta son algo superiores a la media, $24.1 \%$ y $23.6 \%$ respectivamente.

11. Es preciso aclarar que en las distintas tablas la información proporcionada respecto a las ciudades abordadas (Gijón, Oviedo y Avilés) se refiere a los recuentos y porcentajes de cada categoría. De modo que en cada celda se recoge el recuento correspondiente para esa categoría y, a continuación, se señala el porcentaje. En cuanto al resto de localidades, se recoge la media de porcentajes respecto a cada categoría $(\chi)$.

12. Fundación Comarcas Mineras para la Formación y Promoción del Empleo, que puede consultarse en su página Web: http://www.fucomi.com 
TABLA 2. Conocimiento de la existencia de becas para aprender oficios.

\begin{tabular}{|l|rr|rr|rr|c|}
\hline \multirow{2}{*}{$\begin{array}{c}\text { ¿Conoces } \\
\text { algún lugar...? }\end{array}$} & \multicolumn{5}{|c|}{ Localidades } & $\begin{array}{c}\text { Otras } \\
\text { Localidades }\end{array}$ \\
\cline { 2 - 9 } & \multicolumn{2}{|c|}{ Avilés } & \multicolumn{2}{|c|}{ Gijón } & \multicolumn{2}{c|}{ Oviedo } & $\chi$ \\
\hline $\mathrm{Si}$ & 27 & $13,7 \%$ & 112 & $23,6 \%$ & 128 & $24,1 \%$ & $21.3 \%$ \\
\hline No & 157 & $79,7 \%$ & 336 & $70,7 \%$ & 383 & $72,1 \%$ & $73.9 \%$ \\
\hline NS/NC & 13 & $6,6 \%$ & 27 & $5,7 \%$ & 20 & $3,8 \%$ & $4.8 \%$ \\
\hline
\end{tabular}

$\mathrm{Al}$ preguntar a los estudiantes que responden afirmativamente al ítem anterior acerca de las becas que conocen, cabe señalar que un $14.5 \%$ de los encuestados no saben o no contestan a esta pregunta, lo que nos indica que el alumnado piensa que deben existir becas aunque son incapaces de concretar su respuesta. Por otro lado, de la multiplicidad de respuestas obtenidas sus principales aportaciones son: becas del Ayuntamiento, becas de empresas, becas de los fondos mineros y del INEM. Existe además, en todas las localidades, un importante grado de confusión, al reseñar algunos alumnos respuestas incorrectas o que no se corresponden exactamente con el sentido de la pregunta formulada, como por ejemplo; obtener matrícula de honor en Bachiller, cursos de protocolo y becas universitarias.

Con el objetivo de seguir ahondando en el conocimiento que el alumnado tiene de diversos programas formativos dirigidos al aprendizaje de oficios, de las que podrían beneficiarse inmediatamente al finalizar la E.S.O., se les pregunta si han oído hablar de las Escuelas Taller, de las Casas de Oficios, de los programas de Garantía Social y de los cursos del $\mathrm{INEM}^{13}$. Como se puede apreciar en la tabla 3, el $71.2 \%$ de los estudiantes manifiesta haber oído hablar de los cursos de formación que proporciona el INEM, el 61.1\% manifiesta conocer la existencia de las Escuelas Taller y el 61\% menciona los Programas de Garantía Social. Por el contrario, las Casas de Oficios resultan las grandes desconocidas, puesto que solamente el $20.8 \%$ de los encuestados hacen referencia de ellas.

Al mismo tiempo, existen diferencias con relación a los programas formativos de los que ha oído hablar el alumnado de Gijón, Oviedo y Avilés:

- Respecto a las Escuelas Taller las diferencias son estadísticamente significativas (Coeficiente de contingencia $=0.089 ; \mathrm{P}=0.008$ ). Avilés con el $60.9 \%$ de estudiantes que han escuchado cuestiones relativas a las Escuelas Taller, se sitúa en torno al porcentaje medio de las demás localidades asturianas $(61.1 \%)$. Sin embargo, en Gijón el porcentaje desciende hasta el 53.3\% y en Oviedo al 48.2\%, situándose a trece puntos de distancia del porcentaje medio. En Gijón se ofertan diez programas, en Avilés nueve, y en Oviedo siete ${ }^{14}$.

- En cuanto a las Casas de Oficio las diferencias son estadísticamente significativas (Coeficiente de contingencia $=0.186 ; \mathrm{P}=0.000$ ). Mientras en Gijón y Oviedo el porcentaje del alumnado que ha oído hablar de este programa se sitúa en torno al

13. No se les pregunta por los Talleres de Empleo al encontrarse lejanos de su futuro inmediato, al ser uno de los requisitos tener 25 años o más y estos jóvenes tienen mayoritariamente dieciséis años.

14. Esta información procede de la página Web del Ministerio de Educación: http://www.mec.es 
20\% (similar al porcentaje medio de las demás localidades asturianas), en Avilés este porcentaje se duplica situándose en un $40.6 \%$. En cierta medida es lógico su desconocimiento si tenemos en cuenta que en Asturias sólo hay una promovida por el Ayuntamiento de Gozón, cuya cercanía a Avilés podría estar relacionada con que el porcentaje de esta ciudad se duplique.

TABLA 3. Programas formativos de los que han oído hablar.

\begin{tabular}{|c|c|c|c|c|c|c|c|c|}
\hline \multirow{2}{*}{\multicolumn{2}{|c|}{ Programas formativos }} & \multicolumn{6}{|c|}{ Localidades } & \multirow{3}{*}{$\begin{array}{c}\begin{array}{c}\text { Otras } \\
\text { Localidades }\end{array} \\
\chi \\
61.1 \%\end{array}$} \\
\hline & & \multicolumn{2}{|c|}{ Avilés } & \multicolumn{2}{|c|}{ Gijón } & \multicolumn{2}{|c|}{ Oviedo } & \\
\hline Escuelas Taller & Si & 120 & $60,9 \%$ & 253 & $53,3 \%$ & 256 & $48,2 \%$ & \\
\hline Escuelas Taller & No & 77 & $39,1 \%$ & 222 & $46,7 \%$ & 275 & $51,8 \%$ & $38.9 \%$ \\
\hline Casas de Oficios & Si & 80 & $40,6 \%$ & 86 & $18,1 \%$ & 108 & $20,3 \%$ & $20.8 \%$ \\
\hline Casas de Oficios & No & 117 & $59,4 \%$ & 389 & $1,9 \%$ & 423 & $79,7 \%$ & $79.2 \%$ \\
\hline Garantía Social & $\mathrm{Si}$ & 107 & $54,3 \%$ & 356 & $74,9 \%$ & 313 & $58,9 \%$ & $61 \%$ \\
\hline Garantía Social & No & 90 & $45,7 \%$ & 119 & $25,1 \%$ & 218 & $41,1 \%$ & $39 \%$ \\
\hline Cursos INEM & $\mathrm{Si}$ & 145 & $73,6 \%$ & 340 & $71,6 \%$ & 377 & $71,0 \%$ & $71.2 \%$ \\
\hline Cursos INEM & No & 52 & $26,4 \%$ & 135 & $28,4 \%$ & 154 & $29,0 \%$ & $28.8 \%$ \\
\hline
\end{tabular}

- Los Programas de Garantía Social también presentan diferencias estadísticamente significativas (Coeficiente de contingencia $=0.177 ; \mathrm{P}=0.000$ ). Mientras en Oviedo el porcentaje del alumnado que se ha enterado de la existencia de estos programas es similar al porcentaje medio de otras localidades asturianas, en torno al $61 \%$, en Gijón y Avilés varía significativamente, situándose en el $54.3 \%$ y 74.9\% respectivamente. Casualmente, en Oviedo sólo se oferta un programa y en Gijón dos, mientras Avilés tiene tres y el resto de las localidades once.

- En lo tocante a los cursos del INEM, el porcentaje de alumnos que ha oído hablar de ellos se sitúa en torno al $71.2 \%$. Se puede afirmar que no existen diferencias significativas entre Gijón, Oviedo y Avilés, ni respecto a las demás localidades asturianas.

Si se estima el conocimiento que el alumnado manifiesta tener de otros participantes en los programas formativos abordados en el ítem anterior, recogido en la tabla 4, se observa que el 59.3\% afirma conocer a alguno, frente a sólo un $33.88 \%$ que muestra la respuesta contraria. En cuanto a las ciudades analizadas, las diferencias entre ellas son estadísticamente significativas (Coeficiente de contingencia $=0.155 ; \mathrm{P}=0.000$ ). El porcentaje de estudiantes ovetenses que responde afirmativamente desciende hasta el 51.6\%, situándose muy por debajo de la media de Gijón y Avilés, así como de las demás localidades asturianas abordadas (59.3\%). En cuanto al porcentaje del alumnado que no contesta, varía significativamente entre las ciudades, situándose en Avilés en un 12.2\%, descendiendo en Oviedo al $9.6 \%$, hasta llegar a un $2.9 \%$ en Gijón. 
TABLA 4. Conocimiento de participantes en los programas formativos abordados.

\begin{tabular}{|l|rr|rr|rr|c|}
\hline \multirow{2}{*}{$\begin{array}{c}\text { ¿Conoces } \\
\text { algún lugar...? }\end{array}$} & \multicolumn{5}{|c|}{ Localidades } & $\begin{array}{c}\text { Otras } \\
\text { Localidades }\end{array}$ \\
\cline { 2 - 9 } & \multicolumn{2}{|c|}{ Avilés } & \multicolumn{2}{|c|}{ Gijón } & \multicolumn{2}{c|}{ Oviedo } & $\chi$ \\
\hline $\mathrm{Si}$ & 116 & $58,9 \%$ & 284 & $59,8 \%$ & 274 & $51,6 \%$ & $59.3 \%$ \\
\hline No & 57 & $28,9 \%$ & 177 & $37,3 \%$ & 206 & $38,8 \%$ & $33.8 \%$ \\
\hline NS/NC & 24 & $12,2 \%$ & 14 & $2,9 \%$ & 51 & $9,6 \%$ & $6.9 \%$ \\
\hline
\end{tabular}

Cuando se pregunta al alumnado acerca de los estudios que se necesitan para participar en los mencionados programas, se observa un gran desconcierto en cuanto a los requisitos académicos que deben cumplirse. En la tabla 5 se puede observar que existe un considerable porcentaje de estudiantes que afirman desconocer los estudios necesarios para participar en las Escuelas Taller (23.5\%), Casas de Oficios (34.2\%), programas de Garantía Social $(22.6 \%)$ y cursos del INEM (28.1\%). Concretamente, los porcentajes en los niveles educativos que consideran necesarios varían según el programa concreto al que nos estemos refiriendo. A la luz de la información proporcionada por el alumnado, se aprecia un importante número de alumnos que tienen una imagen claramente errónea del nivel de estudios exigido, situación que obliga a replantear la necesidad y la dirección de los procesos de orientación que han recibido estos alumnos.

Simultáneamente, existen diferencias, estadísticamente significativas, respecto al conocimiento que tienen los estudiantes de los requisitos académicos para acceder a los diversos programas formativos en las ciudades abordadas (Gijón, Oviedo y Avilés):

- Respecto a las Escuelas Taller las diferencias son significativas (Coeficiente de contingencia $=0.159 ; \mathrm{P}=0.001)$. En Gijón y Avilés el porcentaje del alumnado que manifiesta un conocimiento de los prerrequisitos exigidos es similar al de las demás localidades asturianas $(26.5 \%)$, y en Oviedo este porcentaje desciende al $16.9 \%$.

- En cuanto a las Casas de Oficios las diferencias son significativas (Coeficiente de contingencia $=0.181 ; \mathrm{P}=0.000)$. Mientras el porcentaje medio de otras localidades asturianas ronda el 13.9\%, Gijón y Oviedo se sitúan dos puntos por encima o por debajo, respectivamente. Sin embargo, el conocimiento de los estudios que se precisan para acceder a estos estudios es mayor en Avilés, elevándose hasta el $22.8 \%$.

- En cuanto a los Programas de Garantía Social las diferencias son significativas (Coeficiente de contingencia $=0.199 ; \mathrm{P}=0.000)$. En Oviedo y Avilés los porcentajes reales de conocimiento rondan el 31\%, algo por debajo de la media del resto de las localidades que se sitúan en el 37.7\%. Sin embargo, en Gijón el alumnado conoce mejor los requisitos académicos de acceso, contestando adecuadamente el $47.6 \%$.

- En relación con los cursos del INEM las diferencias son estadísticamente significativas (Coeficiente de contingencia $=0.167 ; \mathrm{P}=0.000$ ). En Avilés el porcentaje del alumnado que conoce los requisitos académicos se sitúa en torno a la media (15.3\%), en Gijón asciende al 19.2\%, y en Oviedo se sitúa por debajo en 10.5\%. 
TABLA 5. Estudios considerados necesarios para participar en diversos programas.

\begin{tabular}{|c|c|c|c|c|c|c|c|c|}
\hline \multirow{2}{*}{\multicolumn{2}{|c|}{ Estudios necesarios... }} & \multicolumn{6}{|c|}{ Localidad } & \multirow{3}{*}{$\begin{array}{c}\begin{array}{c}\text { Otras } \\
\text { Localidades }\end{array} \\
\chi \\
26.5 \%\end{array}$} \\
\hline & & \multicolumn{2}{|c|}{ Avilés } & \multicolumn{2}{|c|}{ Gijón } & \multicolumn{2}{|c|}{ Oviedo } & \\
\hline \multirow{4}{*}{ Ninguno } & Escuela Taller & 50 & $25,4 \%$ & 124 & $26,1 \%$ & & $16,9 \%$ & \\
\hline & Casas de Oficios & 45 & $22,8 \%$ & 74 & $15,6 \%$ & 59 & $11,1 \%$ & $13.9 \%$ \\
\hline & Garantía Social & 62 & $31,5 \%$ & 226 & $47,6 \%$ & 166 & $31,3 \%$ & $37.7 \%$ \\
\hline & Cursos INEM & 28 & $14,2 \%$ & 91 & $19,2 \%$ & 56 & $10,5 \%$ & $15.3 \%$ \\
\hline \multirow{4}{*}{ Superar la ESO } & Escuela Taller & 66 & $33,5 \%$ & 147 & $30,9 \%$ & 189 & $35,6 \%$ & $34.3 \%$ \\
\hline & Casas de Oficios & 40 & $20,3 \%$ & 132 & $27,8 \%$ & 152 & $28,6 \%$ & $27.8 \%$ \\
\hline & Garantía Social & 39 & $19,8 \%$ & 95 & $20,0 \%$ & 126 & $23,7 \%$ & $21 \%$ \\
\hline & Cursos INEM & 57 & $28,9 \%$ & 131 & $27,6 \%$ & 153 & $28,8 \%$ & $27.8 \%$ \\
\hline \multirow{4}{*}{$\begin{array}{l}\text { Bachillerato o } \\
\text { FP }\end{array}$} & Escuela Taller & 6 & $3,0 \%$ & 20 & $4,2 \%$ & 14 & $2,6 \%$ & $2.8 \%$ \\
\hline & Casas de Oficios & 15 & $7,6 \%$ & 22 & $4,6 \%$ & 34 & $6,4 \%$ & $5.9 \%$ \\
\hline & Garantía Social & 11 & $5,6 \%$ & 19 & $4,0 \%$ & 30 & $5,6 \%$ & $5.9 \%$ \\
\hline & Cursos INEM & 25 & $12,7 \%$ & 63 & $13,3 \%$ & 77 & $14,5 \%$ & $14 \%$ \\
\hline \multirow{4}{*}{$\begin{array}{l}\text { Estudios } \\
\text { universitarios }\end{array}$} & Escuela Taller & 0 & $0,0 \%$ & 4 & $0,8 \%$ & 3 & $0,6 \%$ & $0.4 \%$ \\
\hline & Casas de Oficios & 0 & $0,0 \%$ & 7 & $1,5 \%$ & 3 & $0,6 \%$ & $0.9 \%$ \\
\hline & Garantía Social & 0 & $0,0 \%$ & 3 & $0,6 \%$ & 2 & $0,4 \%$ & $1 \%$ \\
\hline & Cursos INEM & 1 & $0,5 \%$ & 9 & $1,9 \%$ & 20 & $3,8 \%$ & $2.7 \%$ \\
\hline \multirow{4}{*}{ No lo sé } & Escuela Taller & 36 & $18,3 \%$ & 128 & $26,9 \%$ & 159 & $29,9 \%$ & $23.5 \%$ \\
\hline & Casas de Oficios & 51 & $25,9 \%$ & 180 & $37,9 \%$ & 189 & $35,6 \%$ & $34.2 \%$ \\
\hline & Garantía Social & 42 & $21,3 \%$ & 95 & $20,0 \%$ & 128 & $24,1 \%$ & $22.6 \%$ \\
\hline & Cursos INEM & 48 & $24,4 \%$ & 139 & $29,3 \%$ & 157 & $29,6 \%$ & $28.1 \%$ \\
\hline \multirow{4}{*}{ No contesta } & Escuela Taller & 39 & $19,8 \%$ & 52 & $10,9 \%$ & 76 & $14,3 \%$ & $12.5 \%$ \\
\hline & Casas de Oficios & 46 & $23,4 \%$ & 60 & $12,6 \%$ & 94 & $17,7 \%$ & $17.3 \%$ \\
\hline & Garantía Social & 43 & $21,8 \%$ & 37 & $7,8 \%$ & 79 & $14,9 \%$ & $12.8 \%$ \\
\hline & Cursos INEM & 38 & $19,3 \%$ & 42 & $8,8 \%$ & 68 & $12,8 \%$ & $12.2 \%$ \\
\hline
\end{tabular}

La importancia de los hechos mencionados es mayor si cabe, si tenemos en cuenta que el $49.9 \%$ del alumnado afirma tener pensados varios proyectos respecto a su futuro profesional, y lo han hecho sin tener una información completa acerca de sus opciones. Si estimamos que la consideración de tener varios proyectos supone también un cierto grado de indecisión, el número de estudiantes que no tienen definido un proyecto de futuro profesional se incrementa, pues habría que añadir el $18.1 \%$ que esta indeciso y el $2.2 \%$ no sabe o contesta. Situación constante en las diversas localidades asturianas, donde sólo el $29.8 \%$ señala que tiene un proyecto concreto. 
Finalmente, con la intención de comprobar la influencia de diversos aspectos en la configuración de su proyecto profesional, se pregunta a los estudiantes acerca de la prioridad que conceden a la orientación escolar en su proceso de toma de decisión vocacional. Así, en torno al $51.1 \%$ del total señala que le concede bastante o mucha importancia a las orientaciones del colegio al decidir lo que estudiarán o en lo que trabajarán una vez finalizada la E.S.O., tanto en Gijón, Oviedo y Avilés, como en las demás ciudades analizadas.

\section{Conclusiones}

Si tenemos en cuenta la falta de información detectada anteriormente en los estudiantes respecto a algunos programas formativos, que un elevado número de ellos no tiene definido su futuro profesional, así como la importancia que concede el $51.1 \%$ a las orientaciones proporcionadas desde el centro al decidir lo que estudiará o en lo que trabajará una vez finalizada la Educación Secundaria Obligatoria, será preciso proporcionar una orientación adecuada en su proceso de toma de decisión vocacional.

Establecer un proyecto de orientación profesional es una tarea fundamental para superar las carencias detectadas. Hay que destacar la urgencia de la distribución de tareas específicas y especializadas, en un contexto donde la orientación profesional se vuelve más compleja. La diversidad de itinerarios, asignaturas y estudios, tanto dentro del Estado como fuera de él así lo requieren, porque sino podría plantearse el riesgo de que los alumnos, padres y profesores se queden sin la orientación que necesitan. Loizaga Latorre (2005) cuando se refiere a las tendencias de futuro de los Departamentos de Orientación señala que la sociedad y el sistema educativo piden, cada vez más, consejo a los expertos y que las decisiones se tomen en equipo. De modo, que la coordinación no sólo se tiene que desarrollar con los miembros del propio Departamento y centro, sino también con agentes externos al centro, como son los equipos de trabajo social y los responsables de organismos y entidades municipales y supramunicipales, con los responsables de centros de procedencia del nuevo alumnado, ..., y con los centros de asesoramiento externo. Es decir, se plantea como imprescindible la coordinación entre los diversos profesionales y entidades que llevan a cabo tareas de orientación.

Si cuanto se ha dicho se inscribe dentro de una perspectiva de colaboración y participación, las actuaciones se vuelven apropiadas para el sujeto o colectividades que reciben la acción, así como para las diversas instituciones y empresas. Por lo demás, este proyecto de orientación profesional requiere una red de apoyo y coordinación entre los centros del servicio público de empleo, los servicios de orientación y formación profesional de los centros educativos, así como otros organismos y entidades. Hacia ésta hay que dirigir la atención para que la información y orientación llegue a sus destinatarios de forma asequible, completa, económica y eficiente. En realidad, al proyecto de orientación, si se le priva de cualquier coordinación entre los centros educativos y ayuntamientos, se vuelve parcializado e incapaz de contribuir al proyecto personal profesional. Será tarea de todos profundizar en la participación y colaboración de diversas instituciones y Administraciones, incluida la local ${ }^{15}$.

15. La relevancia del ámbito local en los procesos de orientación, formación y fomento del empleo, ha sido reconocida en la legislación vigente (Jefatura del Estado, 2002, art. 15.; Jefatura de Estado, 2003, art. 4) y en numerosos documentos del Ministerio de Trabajo y Asuntos Sociales (2004a, 2004b, 2006). 


\section{Referencias bibliográficas}

Bell, D. (1989). El advenimiento de la sociedad post-industrial. Madrid: Alianza.

Bonino, C. (2005). "SCUO/LAV". Torino. En Banco Internacional de Documentos de Ciudades Educadoras: http://www.edcities.ben.es

Bowen, J. (1976). Historia de la educación occidental I. El mundo antiguo. Barcelona: Herder.

Castells, M. (1995). La ciudad informacional. Tecnologías de la información, reestructuración económica y el proceso urbano-regional. Madrid: Alianza.

Comisión Europea (1996). "Green paper. Living and working in the information society: people first". $C O M$ n $^{\circ} 389$, de 22 de julio.

Comisión Europea (1997). "Green paper. Partnership for a new organisation of work". COM n ${ }^{\circ} 128$, de 8 de abril.

Consejo de la Unión Europea (2001). Carta de los derechos fundamentales de la Unión Europea, diciembre de 2000. Luxemburgo: Oficina de Publicaciones Oficiales de las Comunidades Europeas.

Faure, E. (dir.) (1973). Aprender a ser. Madrid: Alianza Editorial - UNESCO.

Ferrando, J. (2005). "Dispositivo Local para la Transición Escuela-Trabajo". En Banco Internacional de Documentos de Ciudades Educadoras: http://www.edcities.bcn.es

Foro Social Mundial (2005). Carta Mundial por el Derecho a la Ciudad. En: http://www.forumsocialmundial.org

Jaeger, W. (1957). Paideia: los ideales de la cultura griega. México: F.C.B.

Jefatura del Estado (2002). "Ley Orgánica 5/2002, de 19 de junio, de las Cualificaciones y de la Formación Profesional". Boletín Oficial del Estado, 147, de 20 de junio.

Jefatura del Estado (2003). "Ley Orgánica 56/2003, de 16 de diciembre, de Empleo”. Boletín Oficial del Estado, 301, de 17 de diciembre.

Loizaga Latorre, F. (2005). "Departamentos de Orientación: un análisis del rol orientador y de las nuevas figuras asesoras". Revista Española de Orientación Psicopedagógica, 16 (1), 47-61.

Ministerio de Trabajo y Asuntos Sociales (2004a). Programa Nacional de Formación Profesional. En http://www.mtas.es/empleo/formacion

Ministerio de Trabajo y Asuntos Sociales (2004b). Plan de acción para el empleo en el Reino de España. En http://www.mtas.es

Ministerio de Trabajo y Asuntos Sociales (2006). Acuerdo para la mejora y el crecimiento del empleo. En http://www.mtas.es

Molina Martín, S. (2006). "La educación como ámbito de intervención de la ciudad". Revista de Ciencias de la Educación, 205, 93-109.

Organización de Naciones Unidas (1948). Declaración Universal de Derechos Humanos. Aprobada y proclamada el 10 de diciembre de 1948.

Organización de Naciones Unidas (2000). Carta Europea de salvaguarda de los Derechos Humanos en la Ciudad. Barcelona: Ajuntament de Barcelona. Drets Civils.

Fecha de recepción: 24-07-06

Fecha de revision: 27-03-08

Fecha de aceptación: 21-05-08 